\title{
Snoring and nocturnal reflux: association with lung function decline and respiratory symptoms
}

\author{
Össur Ingi Emilsson ${ }^{1,2}$, Shadi Amid Hägg ${ }^{1,2}$, Eva Lindberg ${ }^{1,2}$, Karl A. Franklin ${ }^{3}$, \\ Kjell Toren ${ }^{4}$, Bryndis Benediktsdottir ${ }^{5,6}$, Thor Aspelund (107, \\ Francisco Gómez Real ${ }^{8}$, Bénédicte Leynaert ${ }^{9}$, Pascal Demoly ${ }^{10,11}$, \\ Torben Sigsgaard $^{12}$, Jennifer Perret (1) ${ }^{13}$, Andrei Malinovschi ${ }^{14}$, \\ Deborah Jarvis $^{15}$, Judith Garcia-Aymerich (16,17,18, Thorarinn Gislason ${ }^{5,6}$ and \\ Christer Janson ${ }^{1,2}$, on behalf of the European Community Respiratory Health \\ Survey sleep working group
}

\section{ABSTRACT}

Introduction: The study aim was to examine the association of snoring and nocturnal gastro-oesophageal reflux (nGOR) with respiratory symptoms and lung function, and if snoring and/or nGOR associated with a steeper decline in lung function.

Methods: Data from the third visit of the European Community Respiratory Health Survey (ECRHS) was used for cross-sectional analysis. Pre- and post-bronchodilator spirometry was performed, and information on sleep, nGOR and respiratory symptoms was collected $(n=5715)$. Habitual snoring and nGOR were assessed by questionnaire reports. Pre-bronchodilator spirometry from ECRHS I, II and III (20 years follow-up) were used to analyse lung function changes by multivariate regression analysis.

Results: Snoring and nGOR were independently associated with a higher prevalence of wheeze, chest tightness, breathlessness, cough and phlegm. The prevalence of any respiratory symptom was $79 \%$ in subjects with both snoring and nGOR versus $56 \%$ in those with neither $(\mathrm{p}<0.001)$. Subjects with both snoring and nGOR had more frequent exacerbations (adjusted prevalence 32\% versus 19\% among "no snoring, no nGOR", $\mathrm{p}=0.003$ ). Snoring but not nGOR was associated with a steeper decline in forced expiratory volume in $1 \mathrm{~s}$ over 10 years after adjusting for confounding factors (change in $\%$ predicted -5.53 , versus -4.58 among "no snoring", $\mathrm{p}=0.04$ ) and forced vital capacity (change in \% predicted -1.94 , versus -0.99 among "no snoring", $\mathrm{p}=0.03$ ).

Conclusions: Adults reporting both habitual snoring and nGOR had more respiratory symptoms and more frequent exacerbations of these symptoms. Habitual snoring was associated with a steeper decline in lung function over time.

@ERSpublications

Middle-aged adults with habitual snoring have a steeper decline in lung function over 10 years compared to controls. Habitual snorers with nocturnal gastro-oesophageal reflux have a higher prevalence of nocturnal respiratory symptoms than controls. http://ow.ly/YsiK30odcMY

Cite this article as: Emilsson ÖI, Hägg SA, Lindberg E, et al. Snoring and nocturnal reflux: association with lung function decline and respiratory symptoms. ERJ Open Res 2019; 5: 00010-2019 [https://doi.org/10.1183/23120541.00010-2019].

Copyright $\odot$ ERS 2019. This article is open access and distributed under the terms of the Creative Commons Attribution Non-Commercial Licence 4.0. 


\section{Introduction}

Sleep disordered breathing and nocturnal gastro-oesophageal reflux (nGOR) are common and comorbid conditions $[1,2]$, and both have inter-relationships with respiratory symptoms. Snoring is associated with respiratory symptoms such as wheezing and chronic bronchitis [3]. Individuals with both sleep disordered breathing and asthma have poorer sleep quality and a lower nocturnal oxygen saturation [4]. Even though sleep disordered breathing induces airway inflammation and airway remodelling $[5,6]$, it is not known if it causes changes in spirometry over time.

Respiratory symptoms are also commonly associated with nGOR and having persistent nGOR induces various respiratory symptoms. We have previously even found nGOR to associate with rhinosinusitis [7]. However, nGOR by itself is only weakly associated with changes in lung function [8-10].

As nGOR and sleep disordered breathing often co-exist and are linked by an increased prevalence in obese individuals $[11,12]$, it is of interest to study their combined effect on the respiratory tract. There is a stronger association between nGOR and snoring than between nGOR and the apnoea-hypopnoea index [9]. As snoring is even more prevalent in the general population, this makes snoring (a symptom of upper airway resistance) even more interesting in this context. Recent data from a small, cross-sectional study found the combination of snoring and nGOR to associate synergistically with exacerbations of respiratory symptoms, with increased microaspirations of gastric contents being one plausible explanation [8]. Otherwise, data on the respiratory effects of snoring and nGOR in combination are lacking.

The aim of the study was to examine the association of snoring and nGOR with respiratory symptoms and lung function, and to determine whether participants with snoring or nGOR had a steeper decline in lung function over a previous 20-year period.

\section{Methods}

\section{Design and study cohort}

This study is a part of the Ageing Lungs in European Cohorts (ALEC) consortium (www.alecstudy.org), which is a large research collaboration that aims to improve knowledge on risk factors of lung diseases and lung function decline. For the current study, data were used from the European Community Respiratory Health Survey (ECRHS) I, II and III, a prospective, international, population-based cohort study with three separate waves of visits over 20 years $[10,13]$. First, $>18000$ young adults $(20-44$ years old, ECRHS I) were recruited in 1991-1993. Thereafter, two examinations have taken place (at 27-57 years (ECRHS II, 1999-2003) and at 39-67 years (ECRHS III, 2010-2014)). Data for this study were available for 5715 participants from 22 European centres and one centre in Melbourne, Australia. Spirometry at ECRHS III was available from 5156 subjects.

The majority of participants in the ECRHS I were randomly selected from the general population, with an additional subgroup selected based on a positive screening questionnaire on respiratory symptoms [14]. In the current study, 759 (13\%) participants came from the symptomatic sample.

Spirometry was performed on all three occasions, with reversibility testing with an inhaled $\beta$-adrenergic bronchodilator only performed in ECRHS III. Data on nGOR and snoring were only available from ECRHS III (figure 1). Therefore, analysis of lung function decline was stratified by symptoms reported at the end of follow-up.

Data from ECRHS III were used for a cross-sectional analysis on the association between snoring, nGOR and respiratory symptoms. Spirometry results, body mass index (BMI), age and smoking history were

Affiliations: ${ }^{1}$ Respiratory, Allergy and Sleep Research, Uppsala University, Uppsala, Sweden. ${ }^{2}$ Respiratory Medicine and Allergology, Akademiska Sjukhuset, Uppsala, Sweden. ${ }^{3}$ Perioperative Sciences and Surgery, Dept of Surgery, Umeå University, Umeå, Sweden. ${ }^{4}$ Occupational and Environmental Medicine, University of Gothenburg, Gothenburg, Sweden. ${ }^{5}$ Dept of Sleep Medicine, Landspitali, Reykjavik, Iceland. ${ }^{6}$ Faculty of

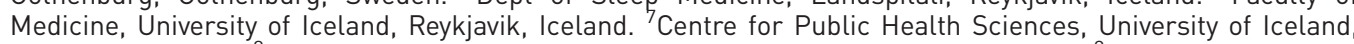
Reykjavik, Iceland. ${ }^{8}$ Dept of Clinical Science, University of Bergen, Bergen, Norway. ${ }^{9}$ Pathophysiology and Epidemiology of Respiratory Diseases, University of Paris, Paris, France. ${ }^{10}$ Dept of Pulmonology, Division of Allergy, Hôpital Arnaud de Villeneuve, University Hospital of Montpellier, Montpellier, France. ${ }^{11}$ Inserm, Sorbonne Université, Equipe EPAR - IPLESP, Paris, France. ${ }^{12}$ Institute of Public Health, Aarhus University, Aarhus, Denmark. ${ }^{13}$ Centre for Epidemiology and Biostatistics, University of Melbourne, Melbourne, Australia. ${ }^{14}$ Clinical Physiology, Uppsala University, Uppsala, Sweden. ${ }^{15} \mathrm{MRC}-\mathrm{PHE}$ Centre for Environment and Health, Imperial College London, London, UK. ${ }^{16}$ ISGlobal, Center for Research in Environmental Epidemiology (CREAL), Barcelona, Spain. ${ }^{17}$ Pompeu Fabra University (UPF), Barcelona, Spain. ${ }^{18} \mathrm{CIBER}$ Epidemiología y Salud Pública (CIBERESP), Barcelona, Spain.

Correspondence: Össur Ingi Emilsson, Lung Dept, Akademiska Sjukhuset, 75185 Uppsala, Sweden. E-mail: ossur.emilssonamedsci.uu.se 


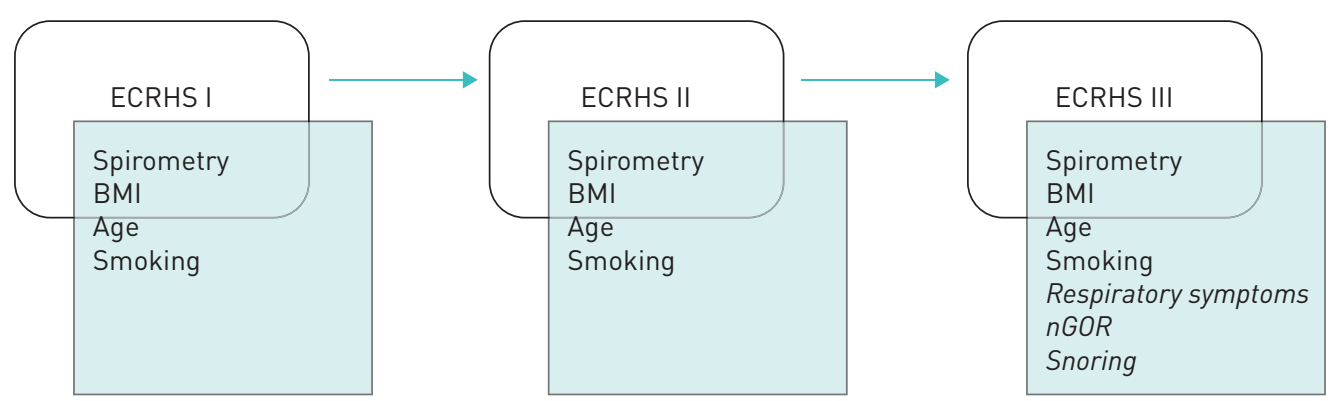

FIGURE 1 Time variant data used from the European Community Respiratory Health Survey (ECRHS) I, II and III. BMI: body mass index; nGOR: nocturnal gastro-oesophageal reflux.

acquired from ECRHS I, II and III for a longitudinal analysis of lung function decline in relation to snoring or nGOR.

\section{Nocturnal gastro-oesophageal reflux}

In ECRHS III, the participants were asked how often during the last 3 months they had experienced heartburn or belching after going to bed. Participants answered on a five-step Likert scale: "Never or almost never", "Less than once a week", "Once or twice a week", "Three to five nights a week" and "Almost every night" [15]. nGOR was defined as reporting heartburn or belching after going to bed once a week or more $[10,16]$.

\section{Snoring}

Snoring was analysed using questions from the Sleep Apnea scale of Sleep Disorders Questionnaire, only posed in ECRHS III [17]. Snoring was first assessed by the question "Have you ever been told that you snore when you sleep?" The $78 \%$ of participants who answered "yes" to that question were further assessed by the following question: "Have you been told that you snore loudly or that your snoring disturbs other people in the last 12 months?" Participants answered on a five step Likert scale: "Never", "Seldom", "Sometimes", "Frequently" and "Every night". Habitual snoring was defined as reporting to snore loudly or disturbingly frequently or every night [18].

\section{Respiratory symptoms}

The questionnaire included questions on respiratory symptoms during the previous 12 months. Symptoms included wheezing, nocturnal chest tightness, breathlessness at rest or after effort, nocturnal breathlessness, nocturnal cough, morning cough and morning phlegm. Exacerbations were defined by a positive answer to the question "In the last 12 months, have you had any episodes/times when your symptoms (cough, phlegm, shortness of breath) were a lot worse than usual?" They also reported the frequency of such exacerbations and "frequent exacerbations" was defined as two or more exacerbations in the previous 12 months. Participants also reported whether they had doctor-diagnosed asthma.

\section{Spirometry}

Lung function in ECRHS III was measured with the spirometer EasyOne (ndd Medical Technologies Inc., Andover, MA, USA) but in ECRHS I and II with the Biomedin Spirometer (Biomedin srl, Padua, Italy). A few centres differed in equipment but without notable heterogeneity in results [19]. Forced expiratory volume in $1 \mathrm{~s}(\mathrm{FEV} 1)$ and forced vital capacity (FVC) were recorded. All subjects performed at least five forced expiratory manoeuvres and maximal values with $\geqslant 200 \mathrm{~mL}$ reproducibility were used for analysis. Predicted values were calculated using data from the Global Lung Function Initiative (GLI) [20].

In ECRHS III, spirometry was performed before and after bronchodilation with $200 \mu \mathrm{g}$ salbutamol. Reversibility in FEV1 was calculated as the difference between pre- and post-bronchodilator FEV1 divided by pre-bronchodilator FEV1.

In ECRHS I and II, spirometry data were recorded similarly; however, no bronchodilation was performed. Predicted values were calculated using data from the GLI.

Change in lung function from ECRHS I to III was calculated both as change in \% of predicted values between the surveys and in millilitres per year, using the actual time between the two spirometries for calculation. 
FIGURE 2 Habitual snoring and nocturnal gastro-oesophageal reflux (nGOR) in the study population.

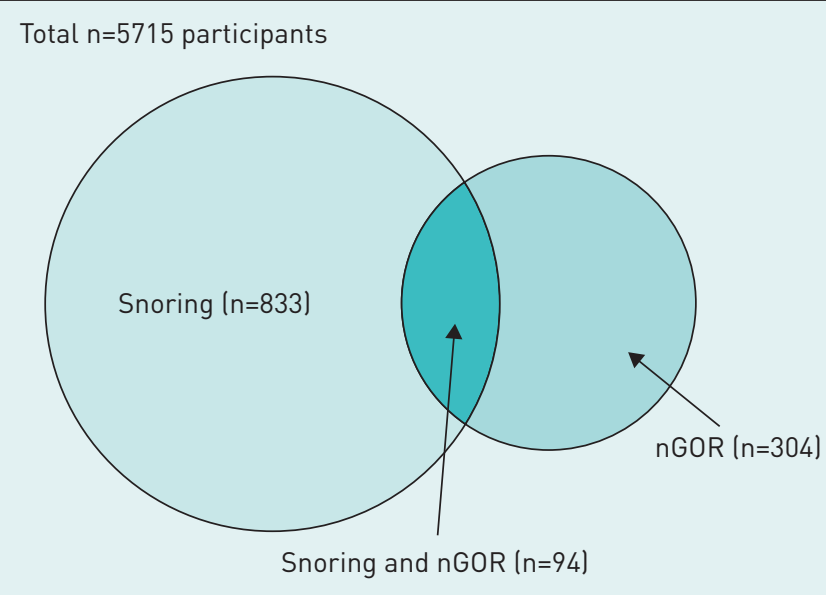

Total $n=5715$ participants

\section{Statistics}

The statistical program STATA (Stata Inc., College Station, TX, USA) version 14.2 was used for statistical analysis. One-way ANOVA, Chi-squared, Wilcoxon rank-sum, multiple linear and logistic regressions were used as appropriate in cross-sectional analysis. Negative binomial regression was used for adjusted analysis of exacerbation frequency. On adjusted cross-sectional analyses of lung function, we adjusted for age, sex, centre, BMI and pack-years. On adjusted analyses of respiratory symptoms, we used the same factors for adjustment (age, sex, centre, BMI and pack-years) and added current smoking status. Lung function changes over time were first analysed by descriptive statistics without adjustments. Lung function changes over time were then analysed by multiple linear regression models, adjusting for the baseline value of the respective lung function parameter, with standard errors clustered by centre [21]. When analysing FEV 1 and FVC, we also adjusted for follow-up time, baseline BMI, change in BMI, pack-years at baseline, change in pack-years, sex and age. When analysing FEV1 and FVC as \% predicted, we did not adjust for sex and age at baseline, as the predicted values were already adjusted for these variables. To validate the results of the multiple linear regression model, we also performed a multilevel mixed linear regression, with centre and subject identifier (ID) as random effect variables (centre hierarchical to subject ID) but otherwise identical adjustments as above.

\section{Results}

The study comprised 5715 participants (2713 men and 3002 women); mean age was $54 \pm 7$ years. Of these, 927 (16\%) participants reported habitual snoring, 398 (7\%) reported nGOR, and 94 (1.6\%) had both snoring and nGOR (figure 2). Participants with nGOR had a higher prevalence of habitual snoring than those without nGOR $(24 \%$ versus $16 \%, \mathrm{p}<0.001)$

Participants reporting either habitual snoring or nGOR were slightly older and had a higher BMI than participants without habitual snoring or nGOR. Habitual snorers were more often ever-smokers and male compared to those without habitual snoring and nGOR (table 1).

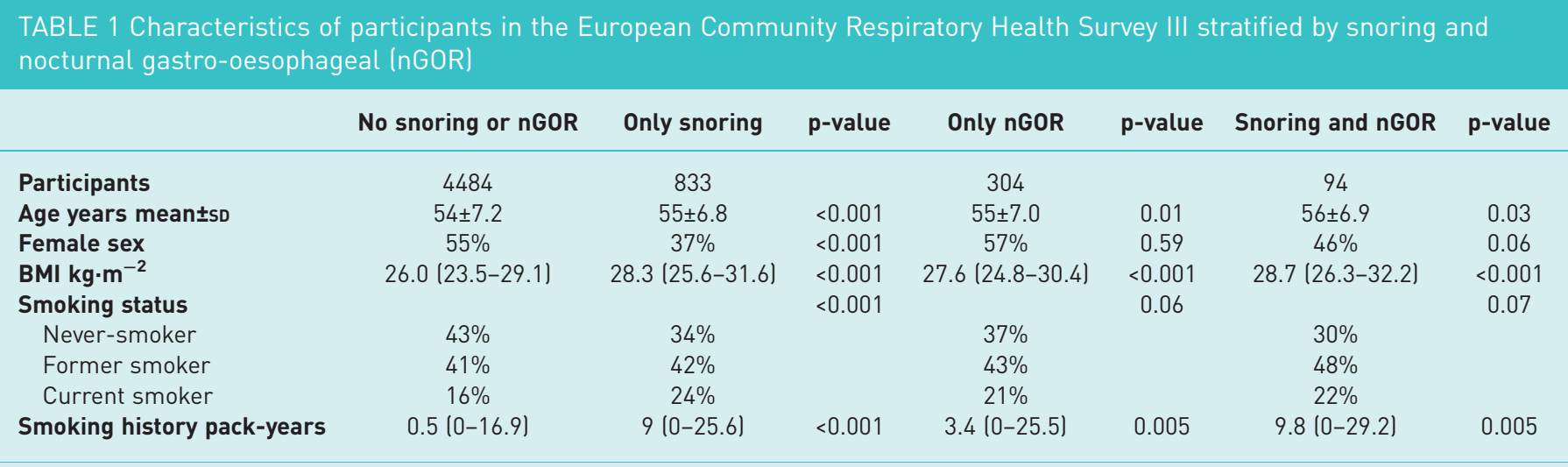

Data are presented as median (interquartile range) unless otherwise stated. p-values were calculated for differences compared to subjects with no snoring or nGOR. BMI: body mass index. 
TABLE 2 Unadjusted analysis of respiratory symptoms by nocturnal gastro-oesophageal reflux (nGOR) and snoring status

\begin{tabular}{|c|c|c|c|c|c|}
\hline & No snoring or nGOR & Only snoring & Only nGOR & Snoring and $n G O R$ & $p$-value \\
\hline Participants & 4484 & 833 & 304 & 94 & \\
\hline Any respiratory symptom & $55.9 \%$ & $68.3 \%$ & $72.3 \%$ & $79.3 \%$ & $<0.001$ \\
\hline Nocturnal chest tightness & $12.5 \%$ & $19.4 \%$ & $23.6 \%$ & $38.0 \%$ & $<0.001$ \\
\hline Breathlessness at rest & $6.4 \%$ & $10.3 \%$ & $12.2 \%$ & $14.9 \%$ & $<0.001$ \\
\hline Breathlessness after effort & $20.7 \%$ & $29.5 \%$ & $29.4 \%$ & $46.7 \%$ & $<0.001$ \\
\hline Morning cough & $10.0 \%$ & $16.2 \%$ & $19.9 \%$ & $24.5 \%$ & $<0.001$ \\
\hline Morning phlegm & $11.5 \%$ & $19.5 \%$ & $17.6 \%$ & $37.6 \%$ & $<0.001$ \\
\hline
\end{tabular}

\section{Cross-sectional symptom analysis}

Overall, respiratory symptoms were more common among participants with habitual snoring and nGOR compared to those without these symptoms. Having both habitual snoring and nGOR had an additive effect on respiratory symptoms (table 2).

The association between habitual snoring (in the absence of nGOR) and respiratory symptoms remained significant after adjustment for BMI, smoking and study centre, and this was also true for the association between nGOR (in the absence of snoring) and respiratory symptoms (table 3).

\section{Exacerbations: cross-sectional}

Among participants reporting respiratory symptoms, the prevalence of frequent exacerbations was significantly higher among participants with the combination of both habitual snoring and nGOR compared to those without either of these symptoms (30\% versus $19 \%, \mathrm{p}=0.02)$ (figure 3). In addition, after adjusting for potential confounding factors, those with both habitual snoring and nGOR were significantly more likely to have frequent exacerbations compared to those without (adjusted prevalence $32 \%$ versus $19 \%, \mathrm{p}=0.003$ ). This association was only seen in males (adjusted prevalence in males $37 \%$

TABLE 3 Adjusted odds of respiratory symptoms and difference in lung function measures by snoring and nocturnal gastro-oesophageal reflux (nGOR)

\section{Only snoring}

$1.35(1.08-1.68)^{*}$

$1.55(1.26-1.89) *$

$1.62(1.33-1.98)^{*}$

$1.33(1.04-1.71)^{*}$

$1.78(1.35-2.37)^{*}$

$1.42(1.19-1.70)^{*}$

$1.36(1.09-1.70)^{*}$

$1.44(1.10-1.88)^{*}$

Morning phlegm ${ }^{\#}$

\section{Spirometry data estimate $(95 \% \mathrm{CI})$}

Pre-bronchodilator FEV1\% pred

Pre-bronchodilator FVC \% pred"

Pre-bronchodilator $\mathrm{FEV}_{1} / \mathrm{FVC}^{+}$

Post-bronchodilator FEV $1 \%$ pred"

Post-bronchodilator FVC \% pred"

Post-bronchodilator $\mathrm{FEV}_{1} / \mathrm{FVC}^{+}$

Reversibility of $F E V_{1} \%{ }^{+}$
Only nGOR

$1.72(1.23-2.39) *$

$2.03(1.44-2.85) *$

$2.00(1.22-3.25)^{*}$

$1.24(1.02-1.50)^{*}$

$2.18(1.40-3.39) *$

$2.14(1.52-3.01) *$

$2.31(1.62-3.28)^{*}$

$1.45(1.03-2.04)^{*}$

$-1.70(-3.85-0.45)$

$-1.37(-3.86-1.12)$

$0.26(-0.30-0.82)$

$-1.54(-3.53-0.46)$

$-1.23(-3.52-1.06)$

$0.16(-0.78-1.11)$

$0.39(-0.33-1.10)$
Snoring and nGOR

$1.47(0.74-2.92)$

$2.95(1.63-5.34)^{*}$

$1.65(0.85-3.18)$

$1.88(1.20-2.95)^{*}$

$3.20(2.17-4.72)^{*}$

$2.68(1.75-4.10)^{*}$

$1.50(0.69-3.25)$

$3.75(2.40-5.84)^{*}$

$-1.11(-5.07-2.86)$

$0.42(-3.17-4.01)$

$-0.44(-2.11-1.24)$

$-0.64(-5.63-4.36)$

$-0.19(-3.52-3.30)$

$-0.59(-3.09-1.91)$

$0.28(-0.88-1.44)$

Without snoring or nGOR as reference group. FEV1: forced expiratory volume in $1 \mathrm{~s}$; FVC: forced vital capacity. \#: adjusted for body mass index (BMI), current smoking status, pack-years, sex, age and centre; ${ }^{\uparrow}$ : adjusted for BMI, pack-years and centre; ${ }^{+}$: adjusted for BMI, pack-years, sex, age and centre. ${ }^{*}$ : $p<0.05$. 
FIGURE 3 Exacerbations of respiratory symptoms. Only calculated for participants reporting any respiratory symptoms. nGOR: nocturnal gastrooesophageal reflux.

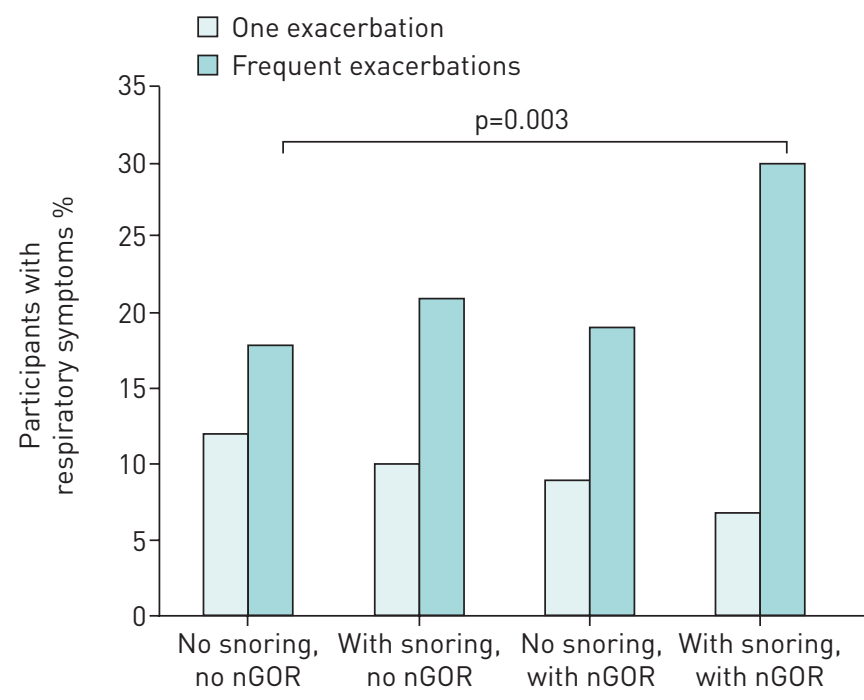

versus $17 \%, \mathrm{p}<0.001$; adjusted prevalence in females $25 \%$ versus $21 \%, \mathrm{p}=0.66$ ). For the whole group, the number of exacerbations reported by participants was significantly higher for those with habitual snoring and nGOR combined compared to those without habitual snoring and nGOR (incidence rate ratio 1.56, 95\% CI 1.06-2.30; $\mathrm{p}=0.006$ ) even after adjusting for confounding factors (predicted number of events 6.4 (95\% CI 3.2-9.6) versus 3.6 (95\% CI 3.2-4.0), $\mathrm{p}=0.02$ ).

\section{Lung function: cross-sectional and changes over time}

In unadjusted cross-sectional analyses, subjects that were habitual snorers had lower lung function levels than subjects without habitual snoring (table 4). Subjects with nGOR had a significantly lower pre-bronchodilator FEV1 than participants without nGOR. However, most of the associations of lung function with snoring and nGOR became statistically nonsignificant after adjustment. The only exception being a significantly higher reversibility of FEV1 after bronchodilator among habitual snorers (table 3 ). Adding asthma diagnosis to the regression model did not affect the increased reversibility among habitual snorers, neither when added as an independent factor nor with an interaction to snoring or nGOR (coefficient for snoring 0.40 ( $\mathrm{p}=0.04)$ and 0.42 ( $\mathrm{p}=0.01)$, respectively).

Complete spirometry data from at least two time-points were available for 4592 subjects. Complete spirometry data from ECRHS II and III were available for 3798 subjects.

FEV1 levels decreased on average $35.4 \mathrm{~mL} \cdot \mathrm{year}^{-1}$ between ECRHS I and ECRHS III for the whole group, regardless of snoring and nGOR status $(n=4592)$. Correspondingly, the FVC decreased on average $27.2 \mathrm{~mL} \cdot$ year $^{-1}$. When stratified by habitual snoring, the group reporting this symptom in ECRHS III was associated with a greater decline in FEV1 and FVC between ECRHS II and ECRHS III, even after adjusting for confounding factors such as BMI and smoking $(n=3798)$ (tables 5 and 6 and figure 4). In these multiple linear regression models, the average effect of snoring can be compared to the average effect of 10 pack-years between ECRHS II and III. The first model finds FEV1 to decline, on average, by $0.9 \%$ due

TABLE 4 Unadjusted analysis of lung function by nocturnal gastro-oesophageal reflux (nGOR) and snoring status

\begin{tabular}{|c|c|c|c|c|c|c|c|}
\hline & No snoring or $n G O R$ & Only snoring & p-value & Only nGOR & p-value & Snoring and nGOR & p-value \\
\hline Participants & 4484 & 833 & & 304 & & 94 & \\
\hline Pre-bronchodilator FEV $1 \%$ pred & $95.4 \pm 14.7$ & $92.2 \pm 14.9$ & $<0.001$ & $93.1 \pm 14.5$ & 0.02 & $92.3 \pm 16.7$ & 0.06 \\
\hline Pre-bronchodilator FEV $1 / F V C \%$ & $75.7 \pm 6.5$ & $75.1 \pm 6.5$ & 0.02 & $75.8 \pm 6.4$ & 0.85 & $74.8 \pm 6.7$ & 0.20 \\
\hline Post-bronchodilator FEV $1 \%$ pred & $97.7 \pm 14.3$ & $95.1 \pm 14.5$ & $<0.001$ & $95.7 \pm 13.9$ & 0.03 & $95.2 \pm 16.2$ & 0.14 \\
\hline Post-bronchodilator FVC \% pred & $98.8 \pm 13.0$ & $96.7 \pm 13.2$ & $<0.001$ & $97.2 \pm 13.3$ & 0.06 & $97.0 \pm 14.2$ & 0.22 \\
\hline
\end{tabular}


TABLE 5 Mean change in lung function over the previous 20 years by snoring and nocturnal gastro-oesophageal reflux (nGOR) at follow-up

No snoring With snoring No nGOR With nGOR

\section{Between ECRHS I and III ( 20 years)}

Change in pre-bronchodilator FEV1 \% pred Change in pre-bronchodilator FEV $1 \mathrm{~mL} \cdot \mathrm{year}^{-1}$ Change in pre-bronchodilator FVC \% pred Change in pre-bronchodilator FVC $\mathrm{mL} \cdot \mathrm{year}^{-1}$ Between ECRHS II and III ( 10 years)

Change in pre-bronchodilator $\mathrm{FEV} 1 \%$ pred Change in pre-bronchodilator FEV 1 mL-year ${ }^{-1}$ Change in pre-bronchodilator FVC \% pred Change in pre-bronchodilator FVC $\mathrm{mL} \cdot$ year $^{-1}$
$-4.66+10.26$

$-34.78 \pm 18.31$

$-0.91 \pm 9.54$

$-26.41 \pm 21.74$

$-4.53 \pm 8.55$

$-41.99 \pm 25.59$

$-0.94 \pm 8.82$

$-32.16 \pm 33.07$

$$
\begin{gathered}
-6.22 \pm 10.54 \\
-38.29 \pm 19.06 \\
-2.57 \pm 9.39 \\
-31.00 \pm 21.68 \\
-6.34 \pm 9.05 \\
-47.75 \pm 27.61 \\
-2.82 \pm 8.91 \\
-39.15 \pm 33.65
\end{gathered}
$$

$-6.08 \pm 11.00$

$-36.89 \pm 20.22$

$-2.04 \pm 9.80$

$-29.93 \pm 22.93$

$-5.43 \pm 8.40$

$-44.41 \pm 26.62$

$-1.69 \pm 8.97$

$-35.43 \pm 32.84$

Data are presented as mean \pm SD. ECRHS: European Community Respiratory Health Survey; FEV1: forced expiratory volume in $1 \mathrm{~s}$; FVC: forced vital capacity.

to snoring and $2.1 \%$ due to a smoking history of 10 pack-years. The second model finds FVC to decline on average $1.0 \%$ from snoring and $1.4 \%$ from 10 pack-years.

A sensitivity analysis, adding doctor-diagnosed asthma to the same regression models, did not affect the results. Habitual snoring associated with a significant decline in FEV1 and FVC in percent predicted between ECRHS II and III ( $\mathrm{p}=0.047$ and $\mathrm{p}=0.037$, respectively).

A multivariable association between nGOR and lung function decline was not seen nor did nGOR have an additive effect on the association between snoring and lung function decline.

\section{Discussion}

Using data from a large, multicentre, population-based cohort, we report a clear association between respiratory symptoms, snoring and nGOR. We found subjects with both nGOR and habitual snoring had the highest frequency of respiratory symptoms. Exacerbations of respiratory symptoms were also more frequent

\begin{tabular}{|c|c|c|c|c|c|c|}
\hline & No snoring & With snoring & $p$-value & No nGOR & With nGOR & p-value \\
\hline \multicolumn{7}{|l|}{$\begin{array}{l}\text { Multiple linear regression, } \\
\text { change between ECRHS I and III } \\
\text { ( } 20 \text { years) }\end{array}$} \\
\hline $\begin{array}{l}\text { Change in pre-bronchodilator } \\
\text { FEV }_{1} \% \text { pred }\end{array}$ & $-4.55(-5.85--3.25)$ & $-5.33(-6.63--4.03)$ & 0.11 & $-4.65(-5.95-3.35)$ & $-5.30(-6.43--4.16)$ & 0.35 \\
\hline $\begin{array}{l}\text { Change in pre-bronchodilator } \\
\text { FVC \% pred }\end{array}$ & $-0.79(-1.89-0.32)$ & $-1.28(-2.60-0.05)$ & 0.16 & $-0.87(-1.98-0.24)$ & $-1.10(-2.65-0.46)$ & 0.72 \\
\hline \multicolumn{7}{|l|}{$\begin{array}{l}\text { Multiple linear regression, } \\
\text { change between ECRHS II and III } \\
\text { ( 10 years) }\end{array}$} \\
\hline $\begin{array}{l}\text { Change in pre-bronchodilator } \\
\mathrm{FEV}_{1} \% \text { pred }\end{array}$ & $-4.58(-5.65--3.52)$ & $-5.53(-6.53--4.53)$ & $0.04^{*}$ & $-4.70(-5.71--3.68)$ & $-5.31(-6.52--4.09)$ & 0.23 \\
\hline $\begin{array}{l}\text { Change in pre-bronchodilator } \\
\text { FVC \% pred }\end{array}$ & $-0.99(-2.07-0.99)$ & $-1.94(-2.81--1.07)$ & $0.03^{*}$ & $-1.10(-2.10--0.10)$ & $-1.47(-2.82--0.13)$ & 0.51 \\
\hline
\end{tabular}
among participants with both nGOR and habitual snoring. We additionally found that habitual snorers had a larger retrospectively measured decline in pre-bronchodilator lung function over the preceding decade.

\section{Respiratory symptoms}

Participants with nGOR and habitual snoring had a higher prevalence of a range of respiratory symptoms, such as wheezing, breathlessness, cough and phlegm, compared to participants without nGOR and

TABLE 6 Adjusted mean change in lung function over the previous 20 years by snoring or nocturnal gastro-oesophageal reflux (nGOR) at follow-up 
a)

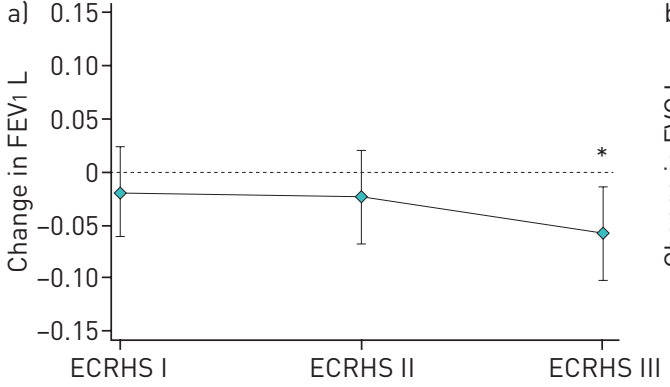

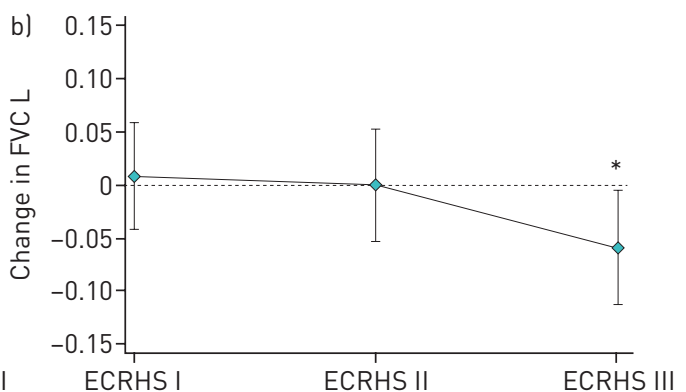
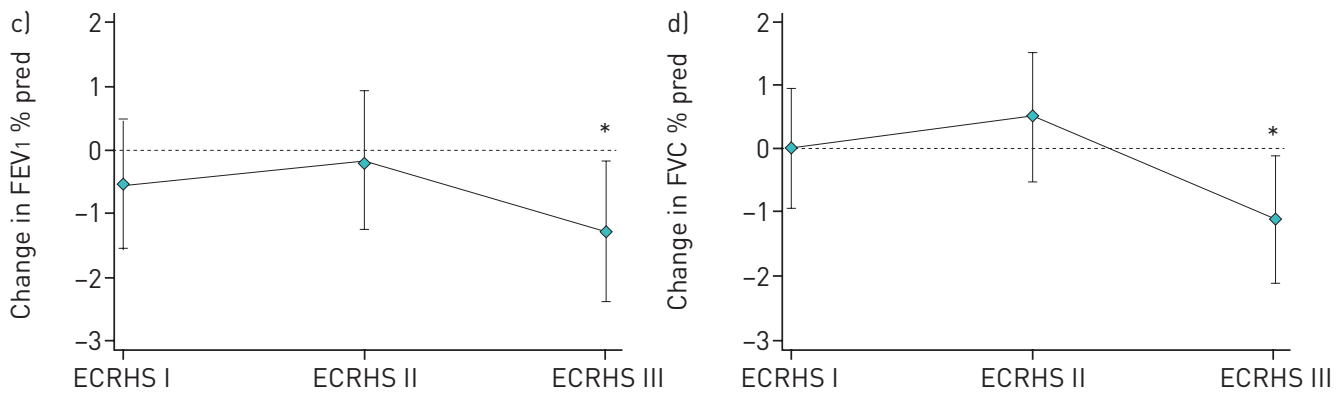

FIGURE 4 The marginal effect of habitual snoring on forced expiratory volume in $1 \mathrm{~s}$ (FEV1) and forced vital capacity (FVC), in terms of a and b) absolute values in litres, and $\mathrm{c}$ and d) \% of predicted, after adjusting for confounding factors such as body mass index, pack-years, centre and follow-up time. For FEV1 and FVC reported as litres, additional adjustments were made for age and sex. Graphs created from a mixed regression model; see the Methods section for details. Error bars represent $95 \%$ confidence intervals. *: p<0.05 compared to European Community Respiratory Health Survey (ECRHS) I.

snoring. After adjustment for confounding factors, the largest difference between the controls and those with both nGOR and snoring was for the sleep-related symptoms: nocturnal chest tightness, waking up from attacks of breathlessness, coughing up phlegm in the morning and waking up from coughing. This predominance of nocturnal symptoms was noteworthy. As daytime symptoms and wheezing had, in total, a relatively similar frequency distribution, it is likely that the nocturnal symptoms do represent a specific phenotypic entity among snorers with nGOR. In addition, previous studies on patients with obstructive sleep apnoea and asthma have found asthma-related quality of life to increase and nocturnal symptoms to diminish after treatment with positive airway pressure [22, 23]. Further studies are needed to better characterise how sleep disordered breathing affects the airways, ideally including more specific markers of sleep disordered breathing and respiratory inflammatory biomarkers.

\section{Exacerbations}

Our previous study on nGOR and snoring in a smaller population, where snoring was objectively measured and nGOR assessed by a validated questionnaire, found the combination of nGOR and snoring to be associated with frequent exacerbations [8]. In the current multicentre population study, which defined snoring using questionnaire data, we confirmed this finding: having two or more exacerbations in a year was more than twice as common for participants with both nGOR and snoring when compared to those with neither. Obstructive sleep apnoea is an independent risk factor for exacerbations among asthma patients [24], and in a previous study we found pepsin in exhaled breath condensate to be increased among subjects with nGOR, as well as snoring and exacerbations of respiratory symptoms [8]. These findings support that nGOR and snoring combined may increase the risk of episodes of worsened respiratory symptoms, possibly through microaspirations of gastric contents. Our study adds that this effect seems to be important at the general-population level. Together, this implies that patients suffering from respiratory symptoms with episodic worsening should be evaluated for potential nGOR and sleep disordered breathing, and treated accordingly.

\section{Lung function}

In the current study, habitual snoring, but not nGOR, was associated with a significant decline in lung function in the preceding decade. This effect was mainly seen between ECRHS II and III, without a significant difference between ECRHS I and II. This is not surprising, as sleep disordered breathing usually increases with increasing age. This means the adverse effects of snoring should presumably be greatest 
under the last years of the study $[25,26]$. Previous studies have found increased airway inflammation and effects on airway remodelling among subjects with obstructive sleep apnoea, and treatment with positive airway pressure may diminish the airway inflammation. This makes it plausible that sleep disordered breathing may affect the lung function $[6,27]$. Our study adds that even habitual snoring in the general population may have detrimental effects on lung function over time. The significant decrease in FEV 1 and FVC may be clinically meaningful and warrants further assessment.

\section{Strengths and weaknesses}

This study's main strengths lie in the well characterised and large general population cohort, with a long follow-up time. In addition, spirometry performed in the same formal manner over 20 years with at least five forced expiratory manoeuvres give further strength to the data. However, the study also has some weaknesses. First, the definition of nGOR is only based on a single question, albeit with answers on a five-point scale. However, reporting nGOR symptoms once a week or more is generally considered sufficient for subject identification $[28,29]$. Regarding the analysis of changes in lung function over time, we were limited by only having data on nGOR and snoring status in ECRHS III, making the temporal relationship the opposite of the study hypothesis. However, as snoring and nGOR are conditions that often develop over a long time, it could be argued that a significant proportion may have been symptomatic over a long time. Indeed, we found that the lung function decline was greater in the second half of the study period, when snoring and nGOR were likely more pronounced. The spirometry in ECRHS I and II were only performed pre-bronchodilator, so reversibility could not be assessed over time.

\section{Conclusion}

Middle-aged adults reporting both nGOR and habitual snoring had more respiratory symptoms, and especially nocturnal respiratory symptoms, than controls. They also had more exacerbations of respiratory symptoms. Those who were habitual snorers shared greater lung function decline over the previous 10 years.

Conflict of interest: Ö.I. Emilsson has nothing to disclose. S.A. Hägg has nothing to disclose. E. Lindberg has nothing to disclose. K.A. Franklin has nothing to disclose. K. Toren has nothing to disclose. B. Benediktsdottir has nothing to disclose. T. Aspelund has nothing to disclose. F. Gómez Real has nothing to disclose. B. Leynaert has nothing to disclose. P. Demoly has nothing to disclose. T. Sigsgaard has nothing to disclose. J. Perret has nothing to disclose. A. Malinovschi has nothing to disclose. D. Jarvis reports grants from the European Union and the Medical Research Council during the conduct of the study. J. Garcia-Aymerich reports payment to her institution for consulting and lectures from AstraZeneca, and lecture fees from Esteve and Chiesi, outside the submitted work. T. Gislason has nothing to disclose. C. Janson has nothing to disclose.

Support statement: The ALEC Study is funded by the European Union's Horizon 2020 Research and Innovation programme under grant agreement number 633212. Funding information for this article has been deposited with the Crossref Funder Registry.

\section{References}

1 Demeter P, Pap A. The relationship between gastroesophageal reflux disease and obstructive sleep apnea. J Gastroenterol 2004; 39: 815-820.

2 Ing AJ, Ngu MC, Breslin AB. Obstructive sleep apnea and gastroesophageal reflux. Am J Med 2000; 108: Suppl. 4a, 120S-125S.

3 Larsson LG, Lindberg A, Franklin KA, et al. Symptoms related to obstructive sleep apnoea are common in subjects with asthma, chronic bronchitis and rhinitis in a general population. Respir Med 2001; 95: 423-429.

4 Sundbom F, Janson C, Malinovschi A, et al. Effects of coexisting asthma and obstructive sleep apnea on sleep architecture, oxygen saturation, and systemic inflammation in women. J Clin Sleep Med 2018; 14: 253-259.

5 Kunos L, Lazar Z, Martinovszky F, et al. Overnight changes in lung function of obese patients with obstructive sleep apnoea. Lung 2017; 195: 127-133.

6 Taille C, Rouvel-Tallec A, Stoica M, et al. Obstructive sleep apnoea modulates airway inflammation and remodelling in severe asthma. PLoS One 2016; 11: e0150042.

7 Schioler L, Ruth M, Jogi R, et al. Nocturnal GERD - a risk factor for rhinitis/rhinosinusitis: the RHINE study. Allergy 2015; 70: 697-702.

8 Emilsson OI, Benediktsdottir B, Olafsson I, et al. Respiratory symptoms, sleep-disordered breathing and biomarkers in nocturnal gastroesophageal reflux. Respir Res 2016; 17: 115.

9 Emilsson OI, Bengtsson A, Franklin KA, et al. Nocturnal gastro-oesophageal reflux, asthma and symptoms of OSA: a longitudinal, general population study. Eur Respir J 2013; 41: 1347-1354.

10 Gislason T, Janson C, Vermeire P, et al. Respiratory symptoms and nocturnal gastroesophageal reflux: a population-based study of young adults in three European countries. Chest 2002; 121: 158-163.

11 Jacobson BC, Somers SC, Fuchs CS, et al. Body-mass index and symptoms of gastroesophageal reflux in women. N Engl J Med 2006; 354: 2340-2348.

12 Basoglu OK, Vardar R, Tasbakan MS, et al. Obstructive sleep apnea syndrome and gastroesophageal reflux disease: the importance of obesity and gender. Sleep Breath 2015; 19: 585-592.

13 Fuertes E, Carsin AE, Anto JM, et al. Leisure-time vigorous physical activity is associated with better lung function: the prospective ECRHS study. Thorax 2018; 73: 376-384. 
14 Burney PG, Luczynska C, Chinn S, et al. The European Community Respiratory Health Survey. Eur Respir J 1994; 7: 954-960.

15 Partinen M, Gislason T. Basic Nordic Sleep Questionnaire (BNSQ): a quantitated measure of subjective sleep complaints. J Sleep Res 1995; 4: 150-155.

16 Emilsson OI, Janson C, Benediktsdottir B, et al. Nocturnal gastroesophageal reflux, lung function and symptoms of obstructive sleep apnea: Results from an epidemiological survey. Respir Med 2012; 106: 459-466.

17 Weatherwax KJ, Lin X, Marzec ML, et al. Obstructive sleep apnea in epilepsy patients: the Sleep Apnea scale of the Sleep Disorders Questionnaire (SA-SDQ) is a useful screening instrument for obstructive sleep apnea in a disease-specific population. Sleep Med 2003; 4: 517-521.

18 Teodorescu M, Consens FB, Bria WF, et al. Predictors of habitual snoring and obstructive sleep apnea risk in patients with asthma. Chest 2009; 135: 1125-1132.

19 Chinn S, Jarvis D, Melotti R, et al. Smoking cessation, lung function, and weight gain: a follow-up study. Lancet 2005; 365: 1629-1635.

20 Quanjer PH, Stanojevic S, Cole TJ, et al. Multi-ethnic reference values for spirometry for the 3-95-yr age range: the global lung function 2012 equations. Eur Respir J 2012; 40: 1324-1343.

21 Coffman CJ, Edelman D, Woolson RF. To condition or not condition? Analysing "change" in longitudinal randomised controlled trials. BMJ Open 2016; 6: e013096.

22 Lafond C, Series F, Lemiere C. Impact of CPAP on asthmatic patients with obstructive sleep apnoea. Eur Respir J 2007; 29: 307-311.

23 Ciftci TU, Ciftci B, Guven SF, et al. Effect of nasal continuous positive airway pressure in uncontrolled nocturnal asthmatic patients with obstructive sleep apnea syndrome. Respir Med 2005; 99: 529-534.

24 Wang Y, Liu K, Hu K, et al. Impact of obstructive sleep apnea on severe asthma exacerbations. Sleep Med 2016; 26: $1-5$.

25 Leppanen T, Toyras J, Mervaala E, et al. Severity of individual obstruction events increases with age in patients with obstructive sleep apnea. Sleep Med 2017; 37: 32-37.

26 Franklin KA, Lindberg E. Obstructive sleep apnea is a common disorder in the population-a review on the epidemiology of sleep apnea. J Thorac Dis 2015; 7: 1311-1322.

27 Zhang D, Luo J, Qiao Y, et al. Measurement of exhaled nitric oxide concentration in patients with obstructive sleep apnea: a meta-analysis. Medicine (Baltimore) 2017; 96: e6429.

28 Dent J, El-Serag HB, Wallander MA, et al. Epidemiology of gastro-oesophageal reflux disease: a systematic review. Gut 2005; 54: 710-717.

29 Vakil N, van Zanten SV, Kahrilas P, et al. The Montreal definition and classification of gastroesophageal reflux disease: A global evidence-based consensus. Am J Gastroenterol 2006; 101: 1900-1920. 